\title{
5. THE DISTRIBUTION OF ATOMIC HYDROGEN IN THE GALAXY
}

\author{
H. C. VAN DE HULST \\ University Observatory, Leiden, Netherlands
}

It is my privilege to report here about the investigations of the distribution of neutral hydrogen in the Galaxy. These observations, conducted by the Netherlands Foundation for Radio Astronomy, were made at Kootwijk and reduced at the Leiden Observatory. More than a dozen persons have participated in this work during the past year. Among them are Prof. Oort and myself, but I should mention three in particular: C. A. Muller has perfected the instruments even further and has supervised the observations that were made during $24 \mathrm{hr}$ a day. G. Westerhout has supervised most of the reductions at Leiden and has prepared the map and model of the outer parts of the Galaxy. M. Schmidt has unravelled the situation in the inner parts of the Galaxy, on the basis of his observations made for this purpose.

The observational material gives, after proper corrections for instrumental effects, the intensity as a function of galactic co-ordinates $l, b$, and the frequency $\nu$. We usually scan in $\nu$ at fixed $l$ and $b$, thus recording a line profile. As the frequency shifts must certainly be ascribed to relative velocities $v$ of the atoms and the observer, we can convert to $(l, b, v)$ as co-ordinates. Our final results are presented in the form of graphs giving the density of atomic hydrogen as a function of the three space co-ordinates $(l, b, r)$ or $(x, y, z)$. Obviously, certain assumptions have been made in this reduction. It seems proper to devote this talk to a summary of the main results together with a discussion of the reliability of the assumptions on which they are based.*

We may distinguish four regions of the Galaxy $(R=$ distance to galactic centre):

(a) near the plane, outer parts, $R>8 \mathrm{kpc}$,

(b) near the plane, inner parts, $3<R<8 \mathrm{kpc}$,

(c) near the plane, central parts, $R<3 \mathrm{kpc}$,

(d) up to $2 \mathrm{kpc}$ from the plane, mainly local.

* The results are summarized in greater detail in Radio Astronomy (I.A.U. Symposium no. 4, 1955), papers 4, 5 and 6, by G. Westerhout and M. Schmidt, Cambridge (1957). For complete data see B.A.N. No. 475 (1957). 
The basic assumption made in reducing observations in regions $(a)$ and $(b)$ is that the velocities consist of a systematic circular motion around the galactic centre combined with random, isotropic, cloud velocities. The latter are called cloud velocities as the thermal motions of the atoms are negligible. No attempt is made to see individual clouds; the instrumental dimensions are such that an average is taken over a volume of space that is several hundred parsecs in each of the three dimensions. Further, in reducing observations in $(a)$ and $(b)$, the assumption is made that the angular velocity $\omega(R)$ and the distribution of cloud velocities in one component $f(v)$ are known. Most of the criticisms may enter here. There may be local or systematic deviations from circular motion, or we may have the wrong $\omega(R)$. Such criticisms are good for warning us against over-interpreting the data, but at the moment the following considerations seem to indicate that no serious deviations exist. We find the longitudes at which the rotational velocities change sign at the correct longitudes of centre and anticentre, known from other studies. The function $\omega(R)$ has been determined from the observations themselves using the tangent method. Data from Australia, not available at the time of the Symposium, * indicate a close equality between the functions $\omega(R)$ determined in the southern and the northern sky so that the motion is circular, not spiral. 'Forbidden velocities', e.g. positive velocities in the Perseus quadrant, can be ascribed to cloud motions of the order of $5^{-10} \mathrm{~km} / \mathrm{sec}$ with occasional suggestions of local deviations of a more systematic character, up to $10 \mathrm{~km} / \mathrm{sec}$.

The reason why regions $(a)$ and $(b)$ have been mentioned separately is that in $(a)$ the $r(v)$ relation is unique and in $(b)$ it is not. Here $r$ is the distance to the sun; the distance $R$ to the centre is uniquely determined in both regions. Perhaps we may pass this point as a technicality although it has formed a tough problem in the reductions. As there is no indication of a structural change at $R=8 \mathrm{kpc}$ we can discuss the results of regions $(a)$ and $(b)$ together.

The main results are shown in the map of Fig. I. This is a contour map of the 'projected' densities: at each point $x, y$, the maximum density found on a line in the $z$-direction (normal to the galactic plane) is represented. As these points do not lie exactly in a plane, the map does not represent the true cross-section with any one plane. However, these points determine fairly well what might be called the true galactic plane. It is $I^{\circ} \cdot 5$ below Ohlsson's plane in the centre direction, $I^{\circ} \cdot 3$ below it near $l=0^{\circ}$, about $1^{\circ}$ or $2^{\circ}$ above it near $l=80^{\circ}$, about coincident with Ohlsson's plane near $l=200^{\circ}$, and again $\mathrm{I}^{\circ}$ or $2^{\circ}$ below it near $l=270^{\circ}$.

\footnotetext{
* Letter from F. J. Kerr to M. Schmidt, 27 October I955.
} 


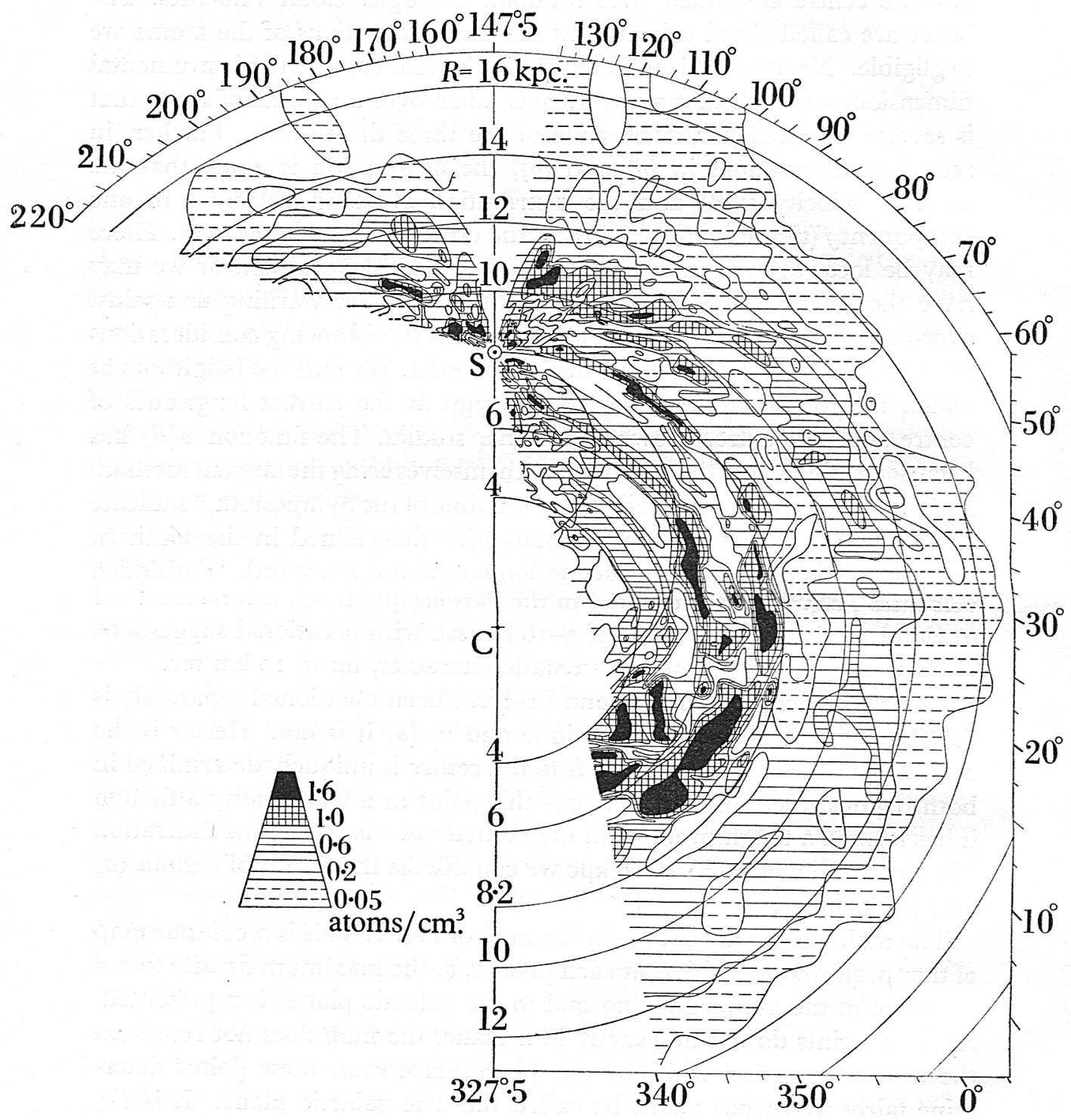

Fig. 1. Maximum hydrogen densities projected on the galactic plane. 
The map shows the same spiral arms that were first plotted three years ago. The well-pronounced arm through the sun is the Orion arm, the heavy arm through the point $l=100^{\circ}, r=2 \mathrm{kpc}$ is the Perseus arm, named after the double cluster near that point, which seems to be its most prominent association. While the coincidence is striking in this projected map, it is not on the three-dimensional model. The maximum hydrogen density in this region is reached at $b=1^{\circ}$ and the suggestion that the main arm is really there is supported by the two strong emission nebulae, IC I 805 and IC 1848 at $b=1{ }^{\circ} 5$. The double cluster is at $b=-3 \cdot 5$, i.e. fully $5^{\circ}$ away and has no emission region on the Palomar Schmidt plates nor $2 \mathrm{I}-\mathrm{cm}$ emission in the Leiden survey. However, local peculiarities are not excluded. This is also illustrated by a splitting of the Perseus arm from $l=65^{\circ}$ to $l=90^{\circ}$.

The arm beyond the Perseus arm is more pronounced in this map than in the published ones as it is about 200 pc above Ohlsson's plane. The maximum density in it is of the order of 0.4 atoms $/ \mathrm{cm}^{3}$, i.e. roughly onequarter of that in the Perseus and Orion arms.

Three heavy arms are seen far from the sun at longitudes between $10^{\circ}$ and $34^{\circ}$. Two are inside $R=8 \mathrm{kpc}$ and one outside. It is quite tempting to call these the extensions of Morgan's Sagittarius arm, Orion arm and Perseus arm, respectively. Perhaps this is correct, but the word spiral arm (and the theories proposed for the origin of spiral arms) is dangerously suggestive. So far, the observations of our own Galaxy do not prove that it is possible to keep track of the identity of a spiral arm after tracing it over more than $60^{\circ}$ of galactocentric longitude. There are plenty of local bulges and deviations and for the present argument it makes little difference whether these are deviations in position or in velocity. For instance, a particularly confused region occurs near $l=30^{\circ}$ to $40^{\circ}$ and I am not certain that it is possible to continue the Perseus arm beyond that point, although another arm takes its (approximate) position.

The densities in the arms between $R=4$ and $8 \mathrm{kpc}$ seem to be slightly higher, systematically, than in those beyond $R=8 \mathrm{kpc}$. This is clearly shown by the map. The cross-sections through the spiral arms resulting from Westerhout's work are, in general, elongated. The diameter of the arm in the plane is of the order of $600 \mathrm{pc}$, while the diameter normal to the plane is closer to 200 or 300 pc. These diameters are measured between points where the density is half the central density. The dimensions appear remarkably constant throughout the region of the Galaxy in which spiral arms have been observed. In this estimate, I have corrected for the slanting direction of the line of sight through the spiral arm. Also, we have 
tried very hard to remove the ellipticity by higher corrections for random cloud motions, but this does not seem possible. The arms seem definitely flattened. There is no theoretical reason against this.

The two other regions of the Galaxy mentioned in my introduction, $(c)$ and $(d)$, have in common line profiles with long wings of low intensity. To observe these wings requires a very stable receiver with a reproducible zero at each frequency. Although it must be more profitable to work with a wide band width on these wings, the present receiver was quite suitable for a reconnaissance.

The preliminary results for the central regions $(R<3 \mathrm{kpc})$ have been published in B.A.N. no. 458 . The wings extend up to 150 or $200 \mathrm{~km} / \mathrm{sec}$ from the zero frequency. In several cases they show up at both the positive and negative velocity side, and they are still present at the centre, where the differential velocities are o. The tentative interpretation is that the central region of our Galaxy contains gas with high turbulent velocities. It has not yet been possible to study the state of rotation of this gas in any detail. Also the provisional continuum measurements seem to indicate a fairly sharp change near $R=3 \mathrm{kpc}$. The region within this distance may be virtually devoid of the sources (ionized gas) that provide the continuous radio emission in the decimetre range in the outer parts. A further study of the intriguing problems connected with the central regions of the Galaxy has been postponed until the new 25-metre telescope is available.

During the last months of measurements at Kootwijk some sample line profiles were measured at points scattered over the sky at latitudes from $30^{\circ}$ to $90^{\circ}$. Half of them show central intensities corresponding to $T=10^{\circ}-$ $25^{\circ}$ near the zero frequency. These peaks may be explained by relatively near clouds. However, they all show extended wings, with temperatures of the order of $2^{\circ}$, i.e. nearly invisible. These wings range on the average from -42 to $+24 \mathrm{~km} / \mathrm{sec}$. The extreme velocities are roughly $50 \%$ larger at either side. The asymmetry is quite pronounced and does not seem to be systematically related to differential galactic rotation. It seems quite likely that these wings are emitted by clouds having velocities of the order of $40 \mathrm{~km} / \mathrm{sec}$ perpendicular to the galactic plane. Such clouds would reach altitudes of the order of $1000 \mathrm{pc}$ and form something intermediate between the disc population and the halo population of the Galaxy. In any case, the gas emitting these wings should not be confused with the gaseous corona of the Galaxy postulated for the interpretation of the background continuum at radio frequencies. The smeared-out density of the gas emitting the $2 \mathrm{I}-\mathrm{cm}$ wings at high latitudes would be of the order of $0 \cdot \mathrm{I}$ atom $/ \mathrm{cm}^{3}$. The systematic velocity of approach is not well understood. The explana- 
tion by a difference in cloud concentration or in ionization between the gas travelling away from the plane and towards the plane does not seem plausible. Perhaps the systematic decrease in angular velocity at these heights may prove a better explanation, although it cannot be the only cause.

\section{Discussion}

W. W. Morgan: Professor van de Hulst's most interesting paper raises an important question of terminology: Are the galactic spiral arms defined uniquely by the $2 \mathrm{I}-\mathrm{cm}$ hydrogen observations? So long as there is sensible agreement in the delineation of the arms by the hydrogen gas and by the blue super-giants, this question is not of much importance; however, in the region of the Double Cluster in Perseus there seems to be a difference; it would appear, therefore, that there might be some justification for distinguishing between 'gas arms' and 'star arms'. The overall agreement between the two seems well established, but the evidence is now rather strong for some local differences.

From the Leiden $21-\mathrm{cm}$ observations it appears that the Double Cluster is located in a region of low hydrogen density. However, in its general neighbourhood a number of early O-stars are observed. (These may have no physical connexion with the Double Cluster itself, but they seem also to be located in a region of low interstellar hydrogen density.) We thus have to explain the presence of a rich concentration of blue giant stars in a region of low interstellar gas density.

The radial velocity measures give strong evidence that the Double Cluster is in the general region in space of the Perseus arm as defined by the $2 \mathrm{I}-\mathrm{cm}$ results. The mean radial velocity for seventeen blue super-giants in the Double Cluster association and for seven red super-giants is close to the $2 \mathrm{I}-\mathrm{cm}$ value for the Perseus arm. In addition, the intensities and velocities of the components of the interstellar lines of $\mathrm{Ca}$ II and $\mathrm{Na}$ I by G. Munch give strong evidence that the Double Cluster is in the general neighbourhood of the Perseus arm.

This question of terminology is especially important at the present time, when, because of limitations in observational resolving power, it is necessary to compare $2 \mathrm{I}-\mathrm{cm}$ results for our Galaxy with optical results for the nearer spirals, like the Andromeda nebula. It would appear to be of the greatest importance to evaluate the degree of accordance of the results of the two methods for our Galaxy.

van de Hulst: There is indeed a conspicuous absence of any $\mathrm{H} \mathrm{I}$ condensation in the region of the $h$ and $\chi$ Persei cluster as indicated in the present survey. However, in general, the agreement with the $\mathrm{H}$ Ir regions, and probably also with the $\mathrm{O}$-associations in these $\mathrm{H}$ in regions, has been very good. Would not Dr Morgan agree that the Double Cluster is a rather exceptional object with very few O-type stars?

Morgan: There are around ten early O-stars in negative galactic latitudes between longitudes $95^{\circ}$ and $107^{\circ}$; it is possible, however, that none of them are directly associated with the Double Cluster. On the other hand, it is exceedingly probable that most of them are concentrated at a distance from the sun similar to that of the Double Cluster. Now, if we omit the $2 \mathrm{I}-\mathrm{cm}$ hydrogen, it also 
appears from the $\mathrm{H}$ II regions themselves that this is a region of low gas density because these $\mathrm{O}$-stars do not have $\mathrm{H}$ Ir regions around them. My only point is that there may be extensive concentrations of early-type stars and even O-stars where there is not a concentration of hydrogen gas.

Bok: I should mention here that Dr Shajn stated very clearly a few years ago that, from his studies at the Grimea Observatory, this region was unusually free of ionized hydrogen.

Spitzer: What is the extension of the hydrogen gas perpendicular to the galactic plane?

Schmidt: The distance between points, where the density is half the density in the plane, is $220 \mathrm{pc}$.

Greenstein: The dust arms do not coincide with the B-star arms in general. This is essentially what Dr Baade pointed out.

Baade: I think that the dust arms and the gas arms in the Andromeda nebula do coincide. They give this picture: where we have $\mathrm{H}$ II regions, we find exactly the same arm with the same extent from these regions and from the dust itself. That is, the $\mathrm{H}$ II regions and the dust arms are exactly co-existent.

Greenstein: I was talking about the star arms. Even within the short span of time for the formation, life and evolution of luminous stars it is very possible that there will be appreciable separation of newly formed objects from the dust and gas regions. I believe it would be over-simplifying the situation we know to exist in our own Galaxy if we said that extra-galactic nebulae have these stars only in dusty regions.

Baade: Well, there, of course, we have the odd situation in Andromeda, that in the inner arms, we essentially see the stars only at the outer fringes because the rest is too obscured. Inside, we see very little, although we do see occasional $\mathrm{H}$ in regions.

de Vaucouleurs: I should like to point out in connexion with Dr Morgan's remarks, that in the Magellanic Clouds, the faint outer arms do not seem to contain strong concentrations of hydrogen gas.

Blaauw: Can anything be said about the systematic differences between the random cloud velocities in different parts of the spiral arms?

van de Hulst: This question of random cloud motions has been a great worry for us because they are needed in the reductions, or everything is blurred in the radial direction. But the data give no information except in those regions where we have what might be called forbidden velocities, in which differential rotation predicts positive velocities though we observe negative tails and conversely. By necessity these regions are quite close to the sun. In these regions there is no definite evidence of systematic differences with distance from the galactic centre. However, perhaps a theoretical argument, due to M. Schmidt, may be used. If the distance scale in the $z$-direction is the same at various distances from the galactic centre and if the gravitational gradient increases strongly, then the velocities must also increase in going towards the centre. This would link up with the fact, which is definitely found from the observations, that quite near the centre, very high velocities are suddenly found. So, perhaps the random cloud velocities increase from a mean of $5 \mathrm{~km} / \mathrm{sec}$, a typical value near the sun, to, say, $10 \mathrm{~km} / \mathrm{sec}$ at $3 \mathrm{kpc}$ from the centre, but we do not know much about it, actually. 